\title{
Modelling Emergence of Money
}

\author{
A.Z. Górski ${ }^{a}$, S. DrożD $\dot{Z}^{a, b}$ AND P. OśWIĘCIMKA ${ }^{a}$ \\ ${ }^{a}$ H. Niewodniczański Institute of Nuclear Physics, Polish Academy of Sciences \\ E. Radzikowskiego 152, 31-342 Kraków, Poland \\ ${ }^{b}$ Faculty of Math. and Natural Sci., University of Rzeszów, 35-310 Rzeszów, Poland
}

\begin{abstract}
The agent-based computational economic (ACE) model with one free parameter (Thresh) proposed by Yasutomi is analyzed in details. We have found that for a narrow range of the parameter, in the money emergence phase, the money lifetime is finite and the "money switching" effect can be observed for long enough time evolution. Long periods of stability are followed by shorter periods with much shorter money lifetimes. Distributions of the money switching points have been found to have non-Cantor distribution on the time axis, i.e. the Rényi exponents determined by the box-counting algorithm equal 1.0 with high accuracy.
\end{abstract}

PACS numbers: 89.65.Gh, 89.75.Fb, 05.45.Tp

\section{Introduction}

The world currency exchange market (FX) dominates other financial markets in volume and range. The FX daily takeover is of order $10^{12}$ USD and transactions take place all over the world, continuously $24 \mathrm{~h}$ a day, 5.5 days a week. The increased interest in analysis of the FX network has started quite recently [1-5]. In this paper, instead of analyzing the present day FX time series we investigate a relatively simple model for emergence and evolution of currency.

About 10000 years ago the neolithic revolution caused essential changes in human life style. In particular, there was a sharp increase in agricultural production and the increased need for exchange of produced goods. The early trade was based on bartering (of livestock, crops etc.). The main obstacle in the barter exchange was the double coincidence of needs, i.e. the necessary condition for a transaction was that both traders should have goods needed by another trader [6]. Due to this obstacle the early trade was relatively modest. Small trade did not stimulate growth of production and consumption, and such situation lasted for a few thousand years. The considerable improvement has happened due to invention of a universal good that was wanted by many traders and that could be easily exchanged for any other good. Such universal good, money, was invented at the end of the second millenium BC. Around 1200 BC the cowry shells (families Cyprraeide and Ovuliadae) were used in China. About $1000 \mathrm{BC}$ the mock cowry shells were introduced and in VIIth century BC the stamped metal coins were in use in Lydia (Turkey). Emergence of money has stimulated growth of trade, production and consumption.

In this paper we study a relatively simple Agent-based Computational Economical (ACE) model for emergence of money of the type introduced by Yasutomi [7]. The model was actually abandoned as leading to stable money, without switching or collapse, and replaced by a more complicated one with larger number of parameters and variables $[7,8]$. In the following we show that this conclusion was premature and for any value of the single parameter of the model (Thresh) one has the money switching effect, i.e. the good playing the rule of money changes from time to time. Some other properties of the model will also be analyzed. This model can be viewed as a toy model that can give us better understanding of the money emergence phenomenon. Even though it cannot give realistic quantitative predictions it provides reasonable insight into quantitative mechanisms. The epistemological rule of simulations as a "third symbol system" (aside verbal and mathematical) has been stressed especially by Ostrom [9].

In the following two sections the model is shortly described and definition of the "universal good" (money) within the model is given. The fourth section is devoted to study the behaviour of the model for different values of the threshold parameter. In the final section our results are summarized.

\section{The model}

In general, an ACE model consists of a number of elements called "agents". The agents can interact with each other (long-range interactions) exchanging, producing and consuming goods according to a set of elementary rules. Serious drawbacks of the traditional economy and important rule that should be played by ACE models in analysis of realistic economical processes has been stressed recently $[10,11]$. ACE models can also be viewed as a generalization of e.g. cellular automata models. At first let us briefly describe the Yasutomi model [7]. 
In the model we have $N$ agents, each agent producing one type of good enumerated by $k=1, \ldots, N$. For the sake of simplicity we assume that the agent number $k$ is producing the good type denoted by $k$. The elementary interaction of two agents ("transaction") consist of several steps including search of the co-trader, exchange of particular goods, change of the agent's buying preferences and finally the production and consumption phase. A sequence of $N$ consecutive transactions is called a turn. In a single turn each of the $N$ agents has chance to take part in exchange of goods, production and consumption.

To each agent (denoted by $k$ ) there are attributed three $N$-dimensional vectors. The possession vector, $P_{i}^{(k)}$, $i=1, \ldots, N$, with non-negative integer components that denote how many units of the $i$-th good has the $k$-th agent, at the moment. The demand vector, $D_{i}^{(k)}$ is actually the "shopping list", i.e. counts how many goods of the type $i$ the agent $k$ is going to buy. Finally, the "world view" vector, $V_{i}^{(k)}$, with non-negative real components is related to the $k$-th agent's shopping preferences. These preferences are evolving with time, depending on the preferences of the other agents (co-traders) as well as according to the success of the previous transaction of the trader. The vector $V_{i}^{(k)}$ is normalized according to

$$
\sum_{i=1}^{N} V_{i}^{(k)}=N, \quad 0 \leq V_{i}^{(k)} \leq N \quad \forall k .
$$

As will be seen below, the higher is the value of $V_{i}^{(k)}$, the agent $k$ is more willing to buy the good $i$. In addition, for each agent there is attributed an integer $w^{(k)}$ equal to the number of the good that the agent urgently needs at the moment and that must be included in the shopping list independently of his preferences $\left(V_{i}^{(k)}\right)$. The other goods at the shopping list will be added depending on the values of the "world view" (preference) vector. In particular, if the value of the component $i$ of the vector $V_{i}$ is greater than the only external parameter of the model, Thresh $\in[0, N]$, the good $i$ will be added to the shopping list. The rule of the parameter Thresh will be discussed later.

Below is the detailed list of steps that constitute a single transaction (as defined in [7]):

Step 1. An agent ("trader") $k$ is chosen randomly.

Step 2. The trader $k$ chooses a co-trader (say, agent $l$ ) who has the largest amount of wanted good, $w^{(k)}$.

Step 3. Both traders check what they have and what they want.

Step 4. The traders exchange their views. At first, they increase the value of component $V_{j}^{(n)}(n=l, k)$ by 1.0 if their previous demands were not satisfied, i.e.

$$
D_{j}^{(n)}>0 \Longrightarrow V_{j}^{(n)} \rightarrow V_{j}^{(n)}+1 \quad(n=l, k) .
$$

Then both traders accept an averaged view:

$$
V_{j}^{(n)} \rightarrow\left[V_{j}^{(k)}+V_{j}^{(l)}\right] / 2 \quad(n=l, k) .
$$

Finally the new views are re-normalized according to the condition (1).
Step 5. The traders create their "shopping list" i.e. they decide what they want to buy. For the trader $k$ :

$$
\text { if } \begin{aligned}
P_{j}^{(l)}>0 \wedge\left(w^{(k)}=j \vee V_{j}^{(k)}>\text { Thresh }\right) \\
\Longrightarrow \quad D_{j}^{(k)}=P_{j}^{(l)} \quad \text { otherwise } D_{j}^{(k)}=0 .
\end{aligned}
$$

The same is done symmetrically $(k \leftrightarrow l)$ by the co-trader $l$ and for all types of goods, $j=1, \ldots, N$.

Step 6. The exchange procedure. The traders "buy" (exchange) goods according to their shopping lists $D_{j}^{(n)}$, where $(n=l, k)$ and $j=1, \ldots, N$. If total amount of goods on both their shopping lists (demands) is identical, $\sum_{j} D_{j}^{(k)}=\sum_{j} D_{j}^{(l)}$, then their demands are fully satisfied and the shopping lists are zeroed.

If the shopping list of one trader (say, $k$ ) is bigger then the shopping list of his co-trader $(l)$ all demands of trader $k$ cannot be satisfied. Hence, after exchange the vector $D_{j}^{(k)}$ will have non-zero components for the goods that could not be bought. In this case the trader with larger shopping list $(k)$ can satisfy his demands partially only. In particular, he selects from his co-trader one unit of good $j$ with the smallest component $D_{j}^{(k)}$ (i.e. the agent prefers to get more rare goods). This procedure is repeated unit by unit until the shopping list $D_{j}^{(l)}$ is zeroed. If one of the traders has empty shopping list (all components are zero) there is no exchange at all and the whole transaction is finished without any exchange. Notice, however, that in spite of this the update of world view vectors was already done (Step 5$)$. Also, during this step the possession vectors $P^{(k)}, P^{(l)}$ of both traders are updated.

Step 7. The final step consists of consumption and production. The traders $k, l$ consume goods specified by the variable $w^{(k)}, w^{(l)}$, respectively. Then, if $P^{(k, l)}=0$ the traders produce one unit of good $k, l$. Finally, we choose new wants for the traders: new values for variables $w^{(k)}, w^{(l)}$ are randomly selected. This ends the elementary transaction process.

The initial conditions are the following: $P_{j}^{(k)}=\delta_{k j}$, $D_{j}^{(k)}=0, V_{j}^{(k)}=1$ and $w^{(k)}$ are chosen randomly from the set $\{1,2, \ldots, N\}$ for each $k$. In particular, the initial shopping list is empty and the "views" of all traders are identical and equally distributed for all goods. In the original version of the model the initial values of the vectors $V^{(k)}$ were set to zero [7], violating the normalization condition (1).

As can be seen from Steps 1-7 the dynamics of the model is partly deterministic, but the random elements are also present and for two runs with identical initial conditions one can obtain different final states. Dynamics of the vector $V^{(j)}$ ("world view") is governed by two effects (Step 4). The first one is due to the "exchange of views". It leads to a kind of herd effect and makes preferences of different agents alike. In addition, in the same step the components of vector $V^{(j)}$ that correspond to the needs that were not satisfied in the previous trans- 
action of the agent $\left(D^{(j)}>0\right)$ are increased. The rule of both these mechanisms is reduced when the parameter Thresh is increased. In particular, for Thresh $=N$ the vectors $V^{(j)}$ become irrelevant for the dynamics and the shopping list is constructed in a purely random way.

Each turn consists of $N$ transations to give opportunity for a transaction for each agent. Clearly, in each transaction do participate two agents and, according to Step 7, the maximum production of goods per turn equals $2 N$.

\section{The notion of money}

Before analysis of the model dynamics we should decide when a given good can be viewed as money. To this end we accept the following natural assumptions for a good to become "money":

(i) Money is the good that is most wanted by all agents, i.e. the expression $\sum_{k} V_{j}^{(k)} / N$ is maximized for the value of $j$ that corresponds to the good that plays the rule of money. The factor $1 / N$ is a normalization constant. We introduce the time dependent function $V_{\max }$ that describes how much the most wanted good (money) is "popular" for the population of all traders,

$$
V_{\max }=\max _{j} \sum_{k} V_{j}^{(k)} / N, \quad 1 \leq V_{\max } \leq N .
$$

(ii) The trade should be nonzero. As will be shown, for some parameter values though there are no exchanges of goods the value of $V_{\max }$ can be quite high.

(iii) In comparison to other goods money should be often exchanged. In particular, the fraction of money exchange should be considerably higher than $1 / N$, the value in the case of equally traded all goods. Also, one can expect a higher level of money supply, i.e. the total amount of money owned by all agents (except the money owned by the money producer).

(iv) The money lifetime should be sufficiently long. If the most wanted good changes ("money switching") from one turn to another one can hardy call such a good "money". Because, in our case, natural unit of time is one turn, we demand that the money lifetime must be $\gg 1$.

To have emergence of money all of the above conditions should be satisfied simultaneously. In the following section we shall investigate behaviour of the model and, especially, emergence of money for different values of the parameter Thresh $\in[0, N]$.

\section{Dynamics of the model}

We take $N=50$ agents to have relatively short computing time to perform many simulations and we start with the highest value of the parameter, Thresh = $N=50$ (clearly, higher values give identical results). Then we shall go down to the value Thresh $=0$.

For large values of the threshold parameter the influence of other agents on a trader (and its "views", i.e. vector $V^{(k)}$ ) is small and his shopping list is dominated by the wanted good $\left(w^{(k)}\right)$. Going down with the threshold we do increase the rule of the "herd effect" in the model.
This herd effect can be viewed either as created by an external global "propaganda" ("marketing") or as an effect of high susceptibility to the behaviour of other agents. Initially, diminishing the parameter Thresh we can observe very small changes in the model behaviour. Even for Thresh $=10$ (20\% of its maximum, only) the model behaviour does not change very much, production and consumption are practically at the zero level. The value of $V_{\text {max }}$ grows from the initial value equal one to about 5 during first 200-300 turns. Hence, this region of the parameter values we call the "starvation phase". In Fig. 1 there are plots for the parameter value Thresh $=8.0$. Clearly, we are still within the starvation phase. For comparison, the dashed lines in Fig. 1a show the values $1+\sqrt{N} / 2$ and $1+\sqrt{N}$, respectively. These values can be viewed as rough estimates for statistical fluctuations in the system with $N$ agents. For sufficiently large values of the parameter Thresh the plot (a) of $V_{\max }$ remains within the area between the dashed lines. The good with highest value of $V_{\max }$ ("money") switches in time, from one good to another ("money switching"). The average money lifetime is below 50 turns. There are long stable periods (the money is fixed for longer than $10^{3}$ turns), as well as periods with very fast money switching (Fig. 1d). However, in the starvation phase there is practically no exchange because the "herd effect" is too weak and the double coincidence of needs blocks trade, production and consumption (Fig. 1b and c). Hence, the conditions (ii) and (iii) for the existence of money are not satisfied. Going down with the parameter Thresh one can slowly increase the rule of the herd effect and stimulate the exchange, production and consumption in the model.

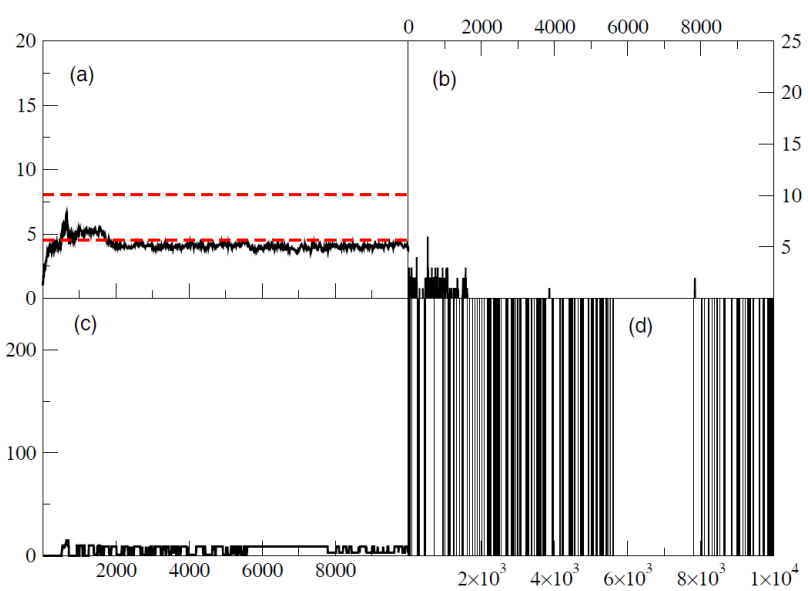

Fig. 1. Model behaviour for the parameter Thresh $=$ 8.0 for first $10^{4}$ turns. (a) Time evolution of $V_{\max }$. The dashed lines shows the values $1+\sqrt{N} / 2$ and $1+\sqrt{N}$, respectively. (b) production of goods; (c) supply of the most wanted good ("money"); (d) vertical lines indicate when the "money switching" takes place for first $10^{4}$ turns.

For Thresh $=7.0$ the value of $V_{\max }$ crosses the upper dashed line (Fig. 2a). More important, production 


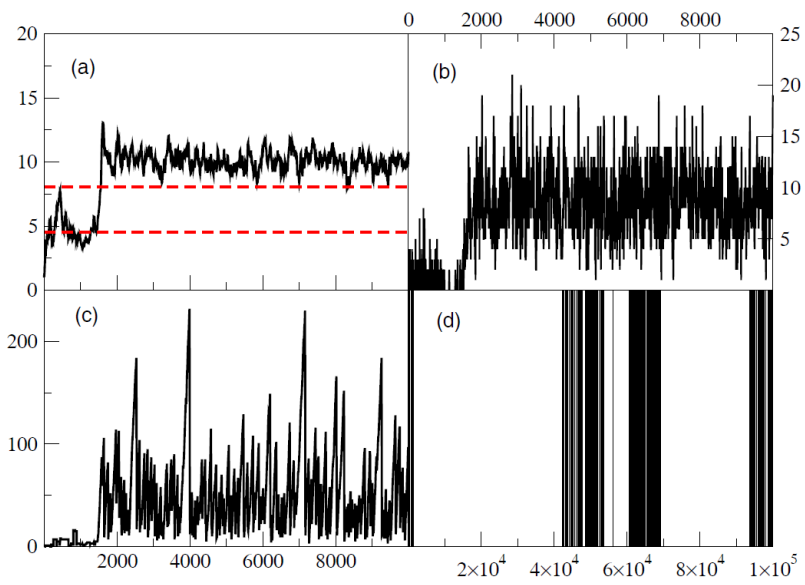

Fig. 2. Model behaviour for the parameter Thresh $=$ 7.0. (a) Time evolution of $V_{\max }$ for first $10^{4}$ turns The dashed lines shows the values $1+\sqrt{N} / 2$ and $1+\sqrt{N}$, respectively; (b) production of goods; (c) supply of the most wanted good ("money"); (d) vertical lines indicate when the "money switching" takes place for first $10^{5}$ turns.

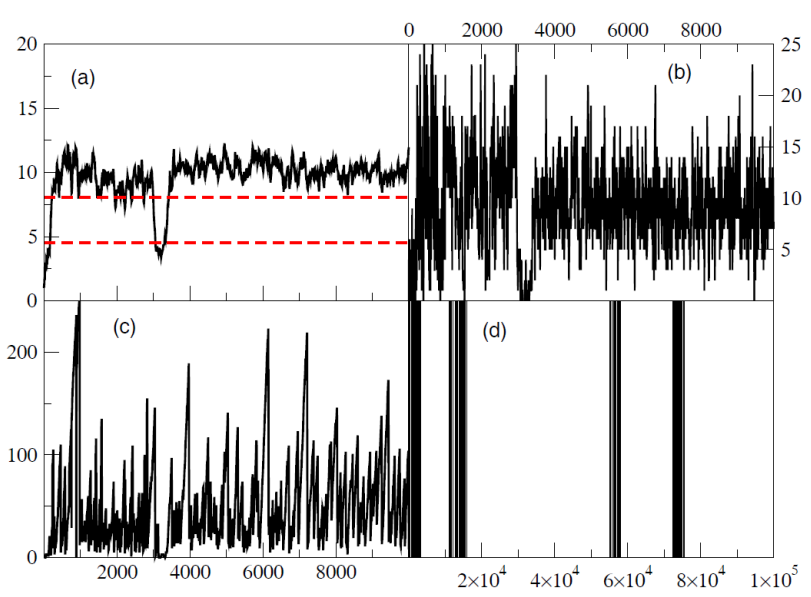

Fig. 3. Model behaviour for the parameter Thresh $=$ 5.0. (a) Time evolution of $V_{\max }$ for first $10^{4}$ turns (the dashed lines as in Fig. 2); (b) production of goods; (c) supply of the most wanted good ("money"); (d) vertical lines indicate when the "money switching" takes place for first $10^{5}$ turns.

of goods (b) and the level of money supply (c) jump substantially. Changes of the product that plays the rule of money are not so frequent. In our simulations we have obtained average money lifetime around 300 turns. It is interesting to notice, that there are quite long periods (of order $>10^{4}$ turns) when the money is stable and periods with much faster changes, with the money lifetime below $10^{2}$ turns (Fig. 2d). Probably, the existence of these long stable periods has misled Yasutomi to the conclusion that the system has reached a stationary state [7]. In addition, money contributes to almost half of all exchanged goods. Hence, we are approaching the second

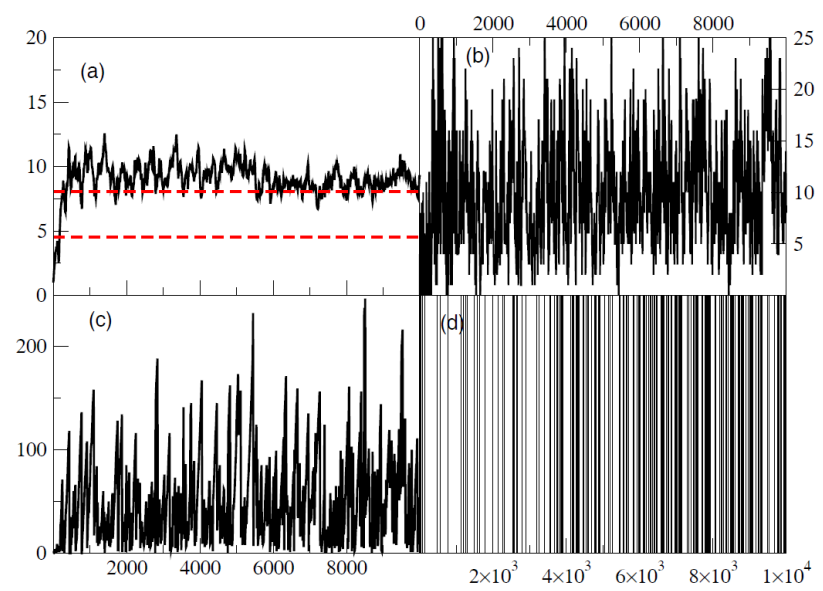

Fig. 4. Model behaviour for the parameter Thresh $=$ 4.0. (a) Time evolution of $V_{\max }$ for first $10^{4}$ turns (the dashed lines as in Fig. 2); (b) production of goods; (c) supply of the most wanted good ("money"); (d) vertical lines indicate when the "money switching" takes place for first $10^{4}$ turns.

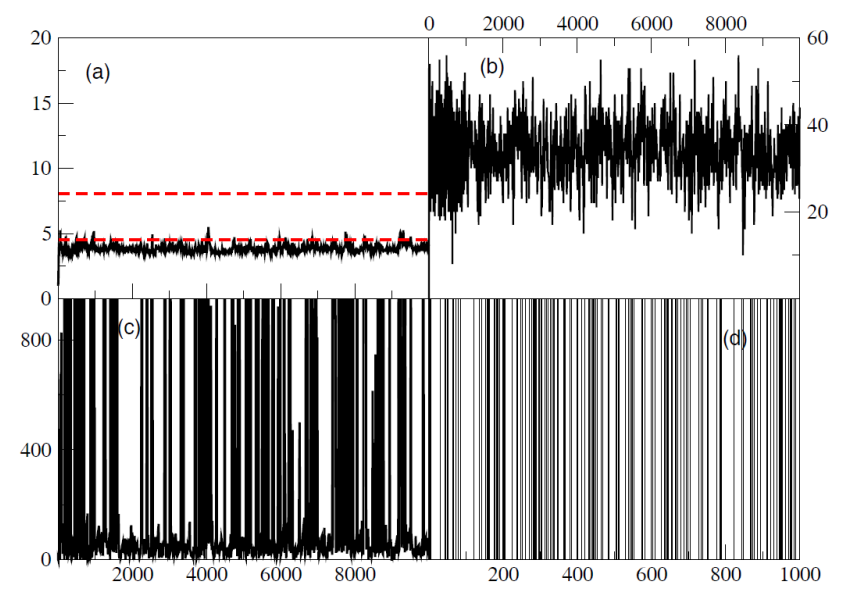

Fig. 5. Model behaviour for the parameter Thresh $=$ 1.0. (a) Time evolution of $V_{\max }$ for first $10^{4}$ turns (the dashed lines as in Fig. 2); (b) production of goods; (c) supply of the most wanted good ("money"); (d) vertical lines indicate when the "money switching" takes place (for the first $10^{3}$ turns).

phase of the model, that we call the "money phase". This phase can be interpreted as a developed market economy. The money phase lasts for a short interval of the parameter Thresh (about a few percent). Similar picture has been obtained for Thresh $=5.0$ (Fig. 3). All simulations were performed up to $10^{6}$ turns. For lucidity, in most of the plots we show the time evolution up to $10^{4}$ turns, as it is qualitatively similar. With exception of the money switching plots (c) with very long stable periods.

For Thresh $=4.0$ we have a qualitative change. The money lifetime becomes about 30 turns, an order of magnitude shorter than for Thresh $=5.0$ (Fig. 4d). Though the values of $V_{\max }$ remain at the similar level (Fig. 4a), 
money contributes to only about a quarter of all exchanged goods, i.e. its rule in the exchanges becomes considerably lower. Hence, we reach the phase where money ceases to exist.

Finally, for smaller values of the parameter the "herd effect" becomes very strong for almost all goods. This results in strong increase of trade, production and consumption. However, money lifetime is very short and traders are willing to exchange basically any good for any other one (Fig. 5). One can call this phase an abundant barter exchange phase. In real economy such phase is rather unusual.

\section{Summary}

We have analyzed the Yasutomi model [7] for different values of the threshold parameter that is responsible for the 'herd effect' in the agents' trading behaviour. For a relatively narrow interval of the parameter (the 'herd effect' neither too strong nor too weak) one can observe emergence of money in the model. Properties of a good playing the rule of money have been defined in Sect. 3. In particular, money becomes the most wanted and the most often traded good. It has been found that the money lifetime is always finite and there is "money switching", i.e. after sufficient time another good overtakes the rule of money in the model. In other words there does not exist an "eternal money".

Also, we have found that the points of money switching do not form a Cantor-type (fractal) set on the time line. Their generalized fractal exponents computed with the box-counting algorithm have been found equal 1.0 with high accuracy.

Generally there are at least three distinct phases in the model. For the large parameter values $(50 \geq$ Thresh $>7$, weak herd effect) the model is in the starvation phase. Production, consumption and exchange of goods is close to zero. The money phase emerges in the region Thresh $\epsilon$ $[5,7]$. In this phase a single good plays the rule of money, i.e. it is often used in exchanges between agents, it has long lifetime and the corresponding component of the vector $V$ is large (greater than $1+\sqrt{N}$ ). Finally, for small values, Thresh $<5$, one has very strong herd effect for many different goods and the abundant barter phase. The agents are exchanging any good for any other good in large amounts and the lifetime of the most wanted good is very short, not much longer than one turn. The dynamics of the system becames purely stochastic. Such behaviour is rather unusual for real markets.

The money lifetime in the money phase can be even longer than $10^{3}$ turns and this can explain why the money switching cannot be observed in the plots of Ref. [7], where the maximum simulation time had this value. However, for long time evolution ( $10^{6}$ turns and more) we found that the long stable periods are followed by shorter periods with much faster money switching (Figs. 3 and 4).

Due to its interesting behaviour the model is worth of more detailed analysis and maybe some modifications would be appropriate. In particular, scaling of the dynamics with the size of the model $(N)$ is not obvious. Further investigations are in progress.

\section{References}

[1] N. Vandewalle, M. Ausloos, Eur. Phys. J. B 4, 257 (1998).

[2] R. Friedrich, J. Peinke, Ch. Renner, Phys. Rev. Lett. 84, 5224 (2000).

[3] M. Ausloos, K. Ivanova, Eur. Phys. J. B 27, 239 (2002).

[4] A.Z. Górski, S. Drożdż, J. Kwapień, P. Oświęcimka, Acta Phys. Pol. B 37, 2987 (2006).

[5] A.Z. Górski, S. Drożdż, J. Kwapień, Eur. Phys. J. B 66, 91 (2008).

[6] C. Menger, Economic Journal 2, 239 (1892), English translation by C.A. Foley.

[7] A. Yasutomi, Physica D 82, 180 (1995).

[8] A. Yasutomi, Chaos 13, 1148 (2003).

[9] T.M. Ostrom, J. Exp. Social Psychology 24, 381 (1988).

[10] J.-P. Bouchaud, Nature 455, 1181 (2008).

[11] J.D. Farmer, D. Foley, Nature 460, 685 (2009). 